\section{Positive impact of the Covid-19 pandemic on dental education}

Published online first on September 18, 2021. DOI: 10.20529/ IJME.2021.069

Keywords: Covid-19, pandemic impact, dental education, teledentistry.ethics

This letter describes some positive effects of the Covid-19 pandemic on dental education in India. The apex body of dental education, the Dental Council of India (DCl) has formulated some universal guidelines based on those of the United States Centers for Disease Control and Prevention, about the use of personal protection and following sterilisation protocols adapted for use in dental clinics, hospitals and academic institutions of dental education in India(1). All individual dental clinics, hospitals and academic institutions have to follow stringent and mandatory sterilisation protocols, hence with the Covid-19 outbreak, some unethical practices of the dental profession such as inadequate sterilisation and improper waste management may be reversed $(1,2)$. Emergency dental procedures also have to follow stringent sterilisation guidelines, to avoid aerosol-generating procedures (1). Dental professionals have been utilising teledentistry facilities during the pandemic which focus on emergency relief of dental pain and infection, and elective planning and scheduling of dental treatment (3).

Postgraduate students, junior residents and lecturers in Indian dental schools have also got ample opportunities for teledentistry consultations, journal clubs, seminars, research write ups, virtual online learning and refreshing the basics. In the pre-Covid-19 era, dental professionals working in government institutions were unable to include problembased learning in their teaching methodology due to multitask management (4). The junior faculty and post graduate students have been getting practical experience in tasks like taking swabs from potential Covid-19 patients, both asymptomatic and symptomatic cases; and in contact tracing, earlier only studied in undergraduate public health textbooks. More emphasis is now placed on the psychological aspects of care, along with virtual learning (5). The most important aspect is that dental professionals are performing productive tasks and are utilising newer avenues of academic learning, as an unexpected positive side effect of the Covid-19 pandemic outbreak.

\section{Conflict of interest and funding: None declared}

Thorakkal Shamim (shamu3duad@gmail.com) Assistant Dental Surgeon, Department of Dentistry, Government Taluk Head Quarters Hospital, Malappuram, 676 519, Kerala INDIA

\section{References}

1. Dental Council of India.COVID-19 guidelines for dental colleges, dental students and dental professionals by Dental Council of India. [Updated 2020 Apr 16; cited 2020 July 27].Available from: http:// www.dciindia.gov.in/Admin/NewsArchives/ DCI\%20Guidelines\%20on\%20COVID-19.pdf

2. Kemparaj VM, Panchmal GS, Kadalur UG. The Top 10 Ethical Challenges in Dental Practice in Indian Scenario: Dentist Perspective. Contemp Clin Dent. 2018 Jan-Mar; 9(1):97-104. Doi:10.4103/ ccd.ccd_802_17.

3. Chopra SS, Sahoo NK. Protocol for teledentistry during COVID-19 in Armed Forces dental establishments. Med J Armed Forces India. 2020 Jul;76(3):356-9. Doi: https://doi.org/10.1016/j.mjafi.2020.05.016

4. Shamim T. Current scenario of problem-based learning in medical and dental education in India. J Educ Ethics Dent. 2017;7(2):49. Doi: 10.4103/ jeed.jeed_5_18

5. Shamim T. Psychological tools in the oral pathology specialty for rapid clinical and histopathological interactive sessions. Med J Armed Forces India. 2021 Jan;77(1): 116. Doi: https://doi.org/10.1016/j.mjafi. 2019.10.001 